\title{
Acid Based Microbicides for the Control of Foodborne and Clinical Pathogens
}

\author{
Tong Zhao* and Govindaraj Dev Kumar \\ Center for Food Safety, College of Agricultural and Environmental Sciences, USA
}

\section{ISSN : 2688-836X}

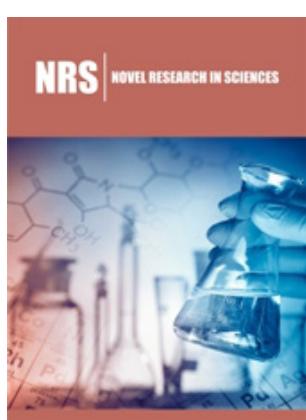

*Corresponding author: Tong Zhao, Center for Food Safety, College of Agricultural and Environmental Sciences, USA

\section{Submission: 淟June 04, 2019 \\ Published: 㘹 June 10, 2019}

Volume 1 - Issue 1

How to cite this article: Tong Z, Govindaraj D K. Acid Based Microbicides for the Control of Foodborne and Clinical Pathogens. Nov Res Sci.1(1). NRS.000503.2019. DOI: $10.31031 /$ NRS.2019.1.000503

Copyright@ Tong Z, This article is distributed under the terms of the Creative Commons Attribution 4.0 International License, which permits unrestricted use and redistribution provided that the original author and source are credited.

\begin{abstract}
Organic acids-based interventions are commonly used by the food industry, especially in poultry and meats for mitigating foodborne pathogens and reducing microbial loads (log reductions). The bactericidal effect of acids is generally caused by its low $\mathrm{pH}$. Acetic, butyric, citric, octanoic, lactic, levulinic, propionic, phenyllactic acid, and acidic calcium sulfate have been shown to have lethality against Gram-positive and Gram-negative microorganisms. Studies have indicated that the applications of acids in different foods have resulted in various log reduction of foodborne pathogens and spoilage microorganisms. However, the efficacy of acid-based interventions is challenged by the development of acid tolerance in bacteria, such as Escherichia coli 0157:H7, which have been implicated in outbreaks associated with acidic foods, such as apple cider, sausages, and tomato ketchup. For pursuing an effective alternative to commonly used acidic sanitizers that is, easy-to-use, cost effective, and environmentally friendly, a microbicide, composed of two Generally Recognized as Safe chemicals, levulinic acid and sodium dodecyl sulfate (SDS) was developed. Its novel bactericidal efficacy ( $>6 \log$ bacteria CFU/ml within $1 \mathrm{~min}$ ) and ability to remove biofilms formed by various human pathogens, including E Coli 0157:H7, Salmonella, Listeria monocytogenes, Streptococcus, and methicillin-resistant Staphylococcus aureus (MRSA) revealed in various studies.
\end{abstract}

Keywords: Pathogens; Intervention; Phenyllactic acid; Antimicrobial activity; Levulinic acid

\section{Introduction}

Recently, organic acid-based intervention procedures have been used in various foods, poultry and meat surface, and food processing facilities for reduction of food borne pathogens and microbial loads. Among them, lactic acid is the acid of choice because it is odorless. The common explanation for its antibacterial activity is the reduction of $\mathrm{pH}$, resulting in a decrease in cytoplasmic $\mathrm{pH}$ of microbial cells by internalization of undissociated acid molecules. In order to keep intracellular $\mathrm{pH}$ at neutrality, bacterial cells have to pump out protons by hydrolyzing ATP, which depletes energy, thereby causing their death eventually. Some acids may produce specific killing effect on pathogens while affecting the organoleptic characteristic of the product.

Octanoic acid has been found to quickly kill inoculated pathogens in food. However, its application in food produces an undesirable odor affecting organoleptic properties of foods and in turn consumer acceptance. Different acids with various antimicrobial activity were combined and determined for their bactericidal effects on E coli 0157:H7, 026:H11, and 0111:NM in rumen content- or feces-contaminated drinking water for cattle. Three combinations, including (a) $0.1 \%$ lactic acid, $0.9 \%$ acidic calcium sulfate, and $0.05 \%$ caprylic acid; (b) $0.1 \%$ lactic acid, $0.9 \%$ acidic calcium sulfate, and $0.1 \%$ sodium benzoate; and (c) $0.1 \%$ lactic acid, $0.9 \%$ acidic calcium sulfate, and $0.5 \%$ butyric acid were evaluated. Results revealed that all three combinations inactivated $>5$ log CFU E coli 0157:H7, 026:H11, and 0111: $\mathrm{NM} / \mathrm{ml}(\mathrm{v} / \mathrm{w})$ in water heavily contaminated with rumen content or feces within 30 min at $21 \mathrm{oC}$. However, their applications are limited to the disinfection of cattle troughs and hardly used as the drinking water because their consumption amounts by cattle as the drink water were significantly lower than the water only group [1].

3-Phenyllactic acid (2-hydroxy-3-phenylpropanoic acid or $\beta$-phenyllactic acid), a derivative of lactic acid is present in honey in higher amount than other phenolic acids. Studies have indicated that phenyllactic acid is present in Thistle Unifloral honey at concentrations of 100 to $800 \mathrm{mg} / \mathrm{kg}$, in Ling Heather honey at $875 \mathrm{mg} / \mathrm{kg}$, and in Manuka honeys at $243 \mathrm{mg} /$ 
kg. Lactic acid bacteria also produce Phenyllactic acid. Dieuleveux et al. [2] first reported its microbicidal effect in Geotrichum candidum. Later, it was confirmed that its antibacterial effect is broad spectrum against various Gram-positive bacteria, including Staphylococcus aureus, Enterococcus faecalis, and Bacillus cereus, and Gram-negative bacteria, including Salmonella and Escherichia coli. In addition to its bactericidal effects, it also inhibits the growth on molds and yeast. We evaluated phenyllactic acid as a sanitizing agent for removal of biofilms produced by either Listeria monocytogenes or Enterobacter cloacae. Results indicated that 1\% phenyllactic acid inactivated more than $6 \log \mathrm{CFU} / \mathrm{ml}$ of inoculated Listeria monocytogenes and E cloacae within $10 \mathrm{~min}$ for planktonic cells at $21^{\circ} \mathrm{C}$. The biofilm elimination assays demonstrated that the killing effect is closely related with the chemical concentrations and exposure time. A concentration of $1 \%$ phenyllactic acid effectively removed early mature biofilm after a 5-min treatment [3]. The studies on the cell membrane permeability through confocal laser scanning microscopy, measurement of extracellular ATP and UVabsorbing substance, membrane potential depolarization, and flow cytometry analysis revealed that the killing mechanism of phenyllactic acid on bacteria is through cell membrane damage and leakage of the intracellular components [4]. Because the fact that lethality to acid adopted $E$ coli $0157: \mathrm{H} 7$ is reduced, as elucidated by its ability to survive in acidic foods, such as apple cider, mayonnaise, and fermented sausage. Food industry demand other effective sanitizers that can be easily adopted into their daily practice. For this purpose, we evaluate many different chemical combinations for their bactericidal effect on various human pathogens. One simple combination with novel antimicrobial activity was created and reported in 2009 by us [5,6]. This combination contains just two chemicals and both of them are classified as Generally Recognized as Safe (GRAS) chemicals, levulinic acid and SDS. Individually, levulinic acid at $0.5 \%$ and SDS at $0.05 \%$ has no bactericidal effect. However, the solution with their combination produces substantially bactericidal effects on all tested microorganisms $(>6$ $\log \mathrm{CFU} / \mathrm{ml}$ within 1 min processing time), including Neisseria gonorrheae, and some spores, mold and yeast.

Its efficacy for controlling foodborne pathogens has been validated by different researchers from various countries, who have broadly evaluated its antimicrobial activity against $E$ coli 0157:H7, Salmonella, Listeria monocytogenes, spores, and virus in produce, poultry, meat, and shrimp through a simple rinse procedure [79]. Their results revealed its application significantly reduced the population of added foodborne pathogens and microbial loads. Its application in seeds for elimination of $E$ coli 0157:H7 and $S$ Typhimurium DT 104 was compared with a treatment of 20,000 ppm of calcium hypochlorite (pH 11.4) or tap water. Similar inactivation rates for $E$ coli 0157:H7 and $S$ Typhimurium were obtained after treatment of alfalfa seeds with $20,000 \mathrm{ppm}$ of calcium hypochlorite and $0.5 \%$ levulinic acid plus $0.05 \%$ SDS (pH 3.0). However, the germination rates of treated seeds were significantly different. A treatment by $0.5 \%$ levulinic acid plus $0.05 \%$ SDS for $1 \mathrm{~h}$ at $21^{\circ} \mathrm{C}$ on alfalfa seeds yielded $80 \%$ germination rate, but the treatment by 20,000 ppm of calcium hypochlorite only yielded $47 \%$ germination rate [10].
The most specific characterization of its application by this novel combination of levulinic acid plus SDS is its strong potential for elimination of biofilm formed by various human pathogens on different materials. For food industry, it demonstrated strong ability to remove E coli 0157:H7, Salmonella, and L monocytogenes in food processing facilities [5,10-14]. For dental industry, it showed fantastic better effects to remove biofilms induced by dental pathogens and microbial loads as a mouthwash than the commercially available mouthwashes. Animal model studies demonstrated that the tissues obtained from gum and mucus after long-term application of this novel bactericide show no pathological difference when compared with water only [15]. For public health, it also demonstrated multi-log reduction on the biofilms formed by methicillin-resistant staphylococcus aureus.

\section{Conclusion}

An acid-based intervention for reduction of food borne pathogens in food industry is broadly applied in the food preservation, poultry, meat, and food processing facilities. The combination through various acids is developed to increase its efficacy to eliminate foodborne pathogens. However, its efficacy is gradually reduced because of appearance of acid-tolerant bacteria, such as E coli 0157:H7. A brand-new bactericide just containing two chemicals was developed. Both chemicals are recommended by US Food and Drug Administration as safe to use, levulinic acid (GRAS, 21 CFR, 172.515) and SDS (as a multipurpose food additive (21 CFR 172.822). Its bactericidal efficacy in food, dental industry, and public health are tested by various studies in the world. Further evaluation, especially in public health, such as to prevent oral sextransmitted diseases as a mouthwash, to eliminate drug-resistant bacteria in hospital environments, as a surface sanitizer is needed for its efficacy validation.

\section{Acknowledgement}

The data presented here were supported by grants from Georgia Department of Agriculture, the State of Georgia's Traditional Industries Program for Food Processing, American Meat Institute Foundation and United States Department of Agriculture-National Institute for Food and Agriculture.

\section{References}

1. Zhao T, Zhao P, West JW, Bernard JK, Cross HG, et al. (2006) Inactivation of Enterhemorrhagic Escherichia Coli in drinking water for cattle contaminated with rumen content or feces. Appl Environ Microbiol 72 (5): 3268-3273.

2. Dieuleveux V, Van der Pyl D, Chataud J, Gueguen M (1998) Purification and characterization of anti-listeria compound produced by Geotrichum candidum. Appl Environ Microbiol 64: 800-803.

3. Liu F, Du L, Zhao T, Zhao P, Doyle MP (2017) Effects of phenyllactic acid as sanitizing agents for inactivation of Listeria monocytogenes biofilms. Food Control 78:72-78.

4. Liu F, Wang F, Du L, Zhao T, Doyle MP, et al. (2018) Antibacterial and antibiofilm activity of phenyllactic acid against Enterobacter cloacae. Food Control 84: 442-448.

5. Zhao T, Zhao P, Doyle MP (2009) Inactivation of salmonella and Escherichia Coli 0157:H7 on lettuce and poultry skin by combination of levulinic acid and sodium dodecyl sulfate. J Food Prot 72(5): 928-936. 
6. Zhao T, Zhao P, Canon J, Doyle MP (2011) Inactivation of salmonella in biofilms, and on chicken cages and preharvest poultry by levulinic acid and sodium dodecyl sulfate. J Food Prot 74 (12): 2024-2030.

7. Cannon JL, Aydin A, Mann AN, Bolton SL, Zhao T, et al. (2012) Efficacy of a levulinic acid plus sodium dodecyl sulfate-based sanitizer on inactivation of human norovirus surrogates. J Food Prot 75(8): 1532-1535.

8. Aydin A, Cannon JL, Zhao T, Doyle MP (2013) Efficacy of a levulinic acid plus sodium dodecyl sulfate (SDS)-based sanitizer on inactivation of influenza A virus on eggshells. Food Environ Virol 5: 215-219.

9. Zhao T, Zhao P, Chen D, Jadeja R, Hung Y, et al. (2014) Reduction of shiga toxin-producing Escherichia Coli and Salmonella typhimurium on beef trim by lactic acid, levulinic acid and sodium dodecyl sulfate treatments. J Food Prot 77(4): 528-537.

10. Zhao T, Zhao P, Doyle MP (2010) Inactivation of Escherichia Coli 0157:H7 and salmonella typhimurium DT 104 on alfalfa seeds by levulinic acid and sodium dodecyl sulfate. J Food Prot 73 (11): 2010-2017.

11. Chen D, Zhao T, Doyle MP (2014) Transfer of foodborne pathogens during mechanical slicing and their inactivation by levulinic acid-based sanitizer on slicers. Food Microbiol 38: 263-269.
12. Chen D, Zhao T, Doyle MP (2015) Single-and mixed-species biofilm formation by Escherichia coli 0157:H7 and Salmonella, and their sensitivity to levulinic acid plus sodium dodecyl sulfate. Food Control 57: 48-53.

13. Chen D, Zhao T, Doyle MP (2015) Control of pathogens in biofilms on the surface of stainless steel by levulinic acid plus sodium dodecyl sulfate. Intern J Food Microbiol 207: 1-7.

14. Chen D, Zhao T (2018) Organic acids, detergents, and their combination for inactivation of foodborne pathogens and spoilage microorganisms: In: Xuetong Fan, Helen Ngo, Changqing Wu (Eds.), Natural and bio-based antimicrobials for food applications. ACS Symposium Series; American Chemical Society, Washington, DC, USA. pp. 63-85.

15. Wang BY, Hong J, Ciancio SG, Zhao T, Doyle MP (2012) A novel formulation effective in killing oral biofilm bacteria. J Intern Academy Periodont $14(3): 56-61$. 